\title{
Caveolin-1 Regulates Human Immunodeficiency Virus-1 Tat-Induced Alterations of Tight Junction Protein Expression via Modulation of the Ras Signaling
}

\author{
Yu Zhong, ${ }^{1}$ Eric J. Smart, ${ }^{2}$ Babette Weksler, ${ }^{4}$ Pierre-Olivier Couraud, ${ }^{5}$ Bernhard Hennig, ${ }^{3}$ and Michal Toborek ${ }^{1}$ \\ ${ }^{1}$ Molecular Neuroscience and Vascular Biology Laboratory, Department of Neurosurgery, ${ }^{2}$ Department of Pediatrics, and ${ }^{3}$ College of Agriculture, University \\ of Kentucky, Lexington, Kentucky 40536, ${ }^{4}$ Weill Medical College of Cornell University, New York, New York 10021, and ${ }^{5}$ Institut Cochin, Institut National \\ de la Santé et de la Recherche Médicale Unité 567, 75014 Paris, France
}

The blood-brain barrier (BBB) is the critical structure for preventing human immunodeficiency virus (HIV) trafficking into the brain. Specific HIV proteins, such as Tat protein, can contribute to the dysfunction of tight junctions at the BBB and HIV entry into the brain. Tat is released by HIV-1-infected cells and can interact with a variety of cell surface receptors activating several signal transduction pathways, including those localized in caveolae. The present study focused on the mechanisms of Tat-induced caveolae-associated Ras signaling at the level of the BBB. Treatment with Tat activated the Ras pathway in human brain microvascular endothelial cells (HBMECs). However, caveolin-1 silencing markedly attenuated these effects. Because the integrity of the brain endothelium is regulated by intercellular tight junctions, these structural elements of the BBB were also evaluated in the present study. Exposure to Tat diminished the expression of several tight junction proteins, namely, occludin, zonula occludens (Z0)-1, and ZO-2 in the caveolar fraction of HBMECs. These effects were effectively protected by pharmacological inhibition of the Ras signaling and by silencing of caveolin-1. The present data indicate the importance of caveolae-associated signaling in the disruption of tight junctions on Tat exposure. They also demonstrate that caveolin-1 may constitute an early and critical modulator that controls signaling pathways leading to the disruption of tight junction proteins. Thus, caveolin-1 may provide an effective target to protect against Tat-induced HBMEC dysfunction and the disruption of the BBB in HIV-1infected patients.

Key words: blood- brain barrier; HIV-1 Tat; Ras; caveolae; caveolin-1; tight junction proteins

\section{Introduction}

Brain microvasculature is actively involved in human immunodeficiency virus-1 (HIV-1) infection of the CNS. HIV-1 crosses the blood-brain barrier (BBB) early in the course of systemic infection and resides in brain macrophages and microglia (Wu et al., 2000; González-Scarano and Martín-García, 2005; Toborek et al., 2005; Banks et al., 2006; Persidsky and Poluektova, 2006). HIV-1 can induce a variety of inflammatory mediators in a variety of cell components of the CNS, including brain endothelial cells. This is important, because HIV-1 trafficking into the brain may be mediated through a "Trojan horse" mechanism, in which HIV-infected $\mathrm{CD}_{4}{ }^{+}$T-lymphocyte and/or circulating monocytes enter the CNS through the BBB via interaction with inflammatory mediators (Wu et al., 2000; González-Scarano and Martín-García, 2005).

During inflammatory responses, leukocytes enter the CNS

Received Jan. 7, 2008; revised June 5, 2008; accepted June 17, 2008.

This work was supported by National Institutes of Health (NIH) Grants MH063022, MH072567, and NS39254. Generation of Tat protein was supported by NIH Grant P01 DA19398.

Correspondence should be addressed to Dr. Michal Toborek, Molecular Neuroscience and Vascular Biology Laboratory, Department of Neurosurgery, University of Kentucky Medical Center, 593 Wethington Building, 900 South Limestone, Lexington, KY 40536. E-mail: michal.toborek@uky.edu.

D01:10.1523/JNEUROSCI.0061-08.2008

Copyright $\odot 2008$ Society for Neuroscience $\quad$ 0270-6474/08/287788-09\$15.00/0 through disrupted tight junctions. Well developed tight junctions are the most prominent feature of the brain endothelium and are responsible for the integrity of the $\mathrm{BBB}$. They limit paracellular flux and restrict permeability across the brain endothelium (Hawkins and Davis, 2005; Abbott et al., 2006). Transmembrane tight junction proteins, such as occludin, are responsible for sealing together brain endothelial cells (Furuse et al., 1993). Transmembrane proteins are linked to the actin cytoskeleton by tight junction accessory proteins, such as zonula occludens (ZO)-1 and ZO-2 (Hawkins and Davis, 2005; Abbott et al., 2006). Disruption of tight junctions is common in HIV-1-infected patients. For example, fragmentation and decreased immunoreactivity for occludin and ZO-1, was detected in the brains of AIDS patients (Dallasta et al., 1999; Boven et al., 2000; Persidsky et al., 2006). These changes were associated with the accumulation of HIV-1infected macrophages, fibrinogen leakage, and apoptosis (Dallasta et al., 1999; Persidsky et al., 2006).

The present study focused on the Ras signaling cascades and its influence on tight junction protein expression. Ras proteins are small GTPases that cycle between inactive GDP-bound and active GTP-bound conformations (Ras-GDP and Ras-GTP, respectively). Several elements of the Ras signaling cascades are localized in caveolae, the dominant type of lipid rafts in endothelial cells (Dykstra et al., 2003; Pike, 2003; Cohen et al., 2004; Rao 
A.

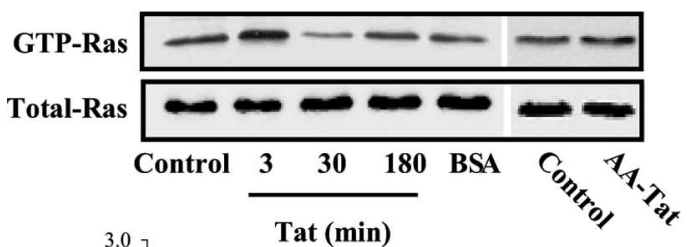

B.

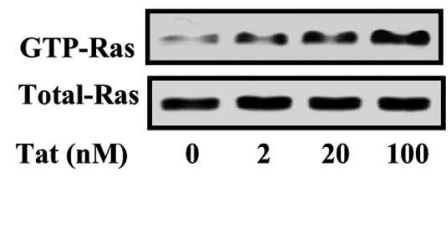

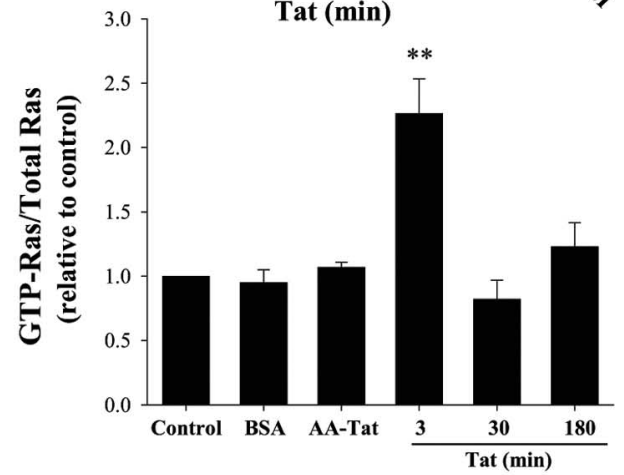

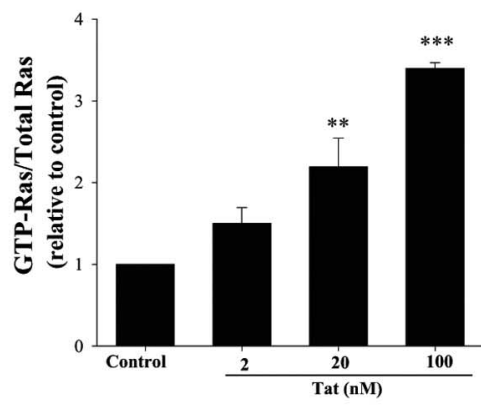

C.

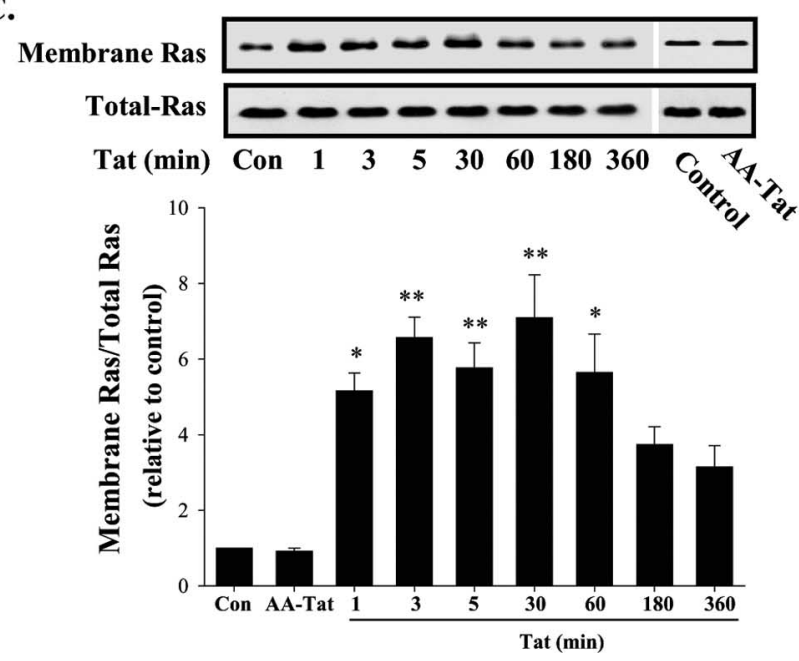

Figure 1. Tat-mediated activation of Ras in HBMECS. $\boldsymbol{A}, \boldsymbol{B}, \mathrm{HBMEC}$ were exposed to Tat for the indicated time points $(\boldsymbol{A})$ or concentration (B). Active GTP-Ras was pulled down from the cell lysates with Raf-1 RBD (Ras binding domain) coupled to glutathione agarose, and Ras protein was determined by immunoblotting with monoclonal anti-Ras antibody. The bottom blots represent total Ras expression in HBMECs. C, The membrane fraction was separated as described in Materials and Methods, and Ras expression was detected using monoclonal anti-Ras antibody. The whole-cell lysate was used to determine the total Ras protein. In A-C, treatment with AA-Tat and/or BSA was used as negative controls. Cells were exposed to AA-Tat or BSA for 3 min. The blots reflect representative data from three different experiments and the bar graphs represent quantified results (mean \pm SD) from these experiments as analyzed by densitometry. ${ }^{*} p<0.05,{ }^{* *} p<0.01$ compared with control cells.

et al., 2004). The principal structural components of caveolae membranes are caveolins, $21-24 \mathrm{kDa}$ integral membrane proteins. Caveolin-1 is the main isoform in endothelial cells and knocking it down eliminates the formation of caveolae (Cohen et al., 2004).

Several vascular effects of HIV-1 observed in the CNS can be reproduced by treatment with HIV-1 protein Tat. Tat is a strong proinflammatory agent that can recruit and induce transendothelial migration of monocytes (Weiss et al., 1999). Tat also affects endothelial cell actin microfilament dynamics, causing actin cytoskeletal rearrangements and disassembly (Wu et al., 2004). Therefore, the aim of the present study was to evaluate the role of caveolae and caveolin-1 in Tat-induced dysfunction of brain endothelial cells. Our novel observations indicate that Tatmediated activation of the Ras signaling is regulated by caveolin-1 in brain endothelial cells. In addition, we demonstrate that inhibition of caveolin-1 and the Ras signaling can attenuate Tat-induced disruption of tight junction proteins.

\section{Materials and Methods}

Cell cultures and treatment. Immortalized human brain microvascular endothelial cells (HBMECs) (Weksler et al., 2005) were cultured in EBM-2 medium, supplemented with EGM-2 SingleQuots (Lonza Walkersville) and 5\% FBS (HyClone). All cell culture dishes were coated with rat tail collagen type 1 (BD Biosciences).

Caveolin-1-deficient $\left(\mathrm{cav}^{-1}{ }^{-/-}\right)$mice were purchased from The Jackson Laboratory and bred through heterozygous mating. These mice were generated in the C57BL/6 genetic background (Razani et al., 2001). Brain microvascular cells were isolated from cav-1 ${ }^{-1-}$ and control C57BL/6 mice as previously described (András et al., 2005). All procedures were approved by the Institutional Animal Care and Use Committee.

HIV Tat protein is released from infected cells and is found circulating in the blood of HIV-1-infected patients (Westendorp et al., 1995; Xiao et al., 2000). Evidence indicates that pathological concentrations of Tat in HIVinfected patients can reach the range of nanograms per milliliter of serum (Xiao et al., 2000). Therefore, cells were treated with Tat at levels between 2 and $100 \mathrm{~nm}$ in the present study. Such concentrations of Tat are consistent with literature data (Rumbaugh et al., 2006; Eugenin et al., 2007).

Tat was produced as described by $\mathrm{Ma}$ and Nath (1997). To determine specificity of Tatinduced effects, Tat solutions were immunoabsorbed using anti-Tat antibody conjugated to protein-A/G plus-agarose (Santa Cruz Biotechnology) and centrifuged to remove precipitated proteins. This form of Tat was called immunoabsorbed Tat (AA-Tat).

Ras GTPase pull-down assay. Ras activity was assayed by using the GTP-Ras pull-down assay according to the manufacturer's instruction (Millipore). Western blots were performed using monoclonal anti-Ras antibody (BD Biosciences Transduction Laboratories).

Caveolin-1 silencing. Caveolin-1 silencing was performed as described previously (Repetto et al., 2005) using a mixture of small interfering RNA (siRNA) corresponding to nucleotides 69-87 of the human caveolin-1 mRNA sequence, $5^{\prime}$-CAUCUACAAGCCCAACAACdTdT-3' (cav-1 siRNA-1), and to nucleotides 223-241, 5' -CCAGAAGGGACACACAGUU-dTdT-3' (cav-1 siRNA-2). Control siRNA was as follows: 5'-AAAGAGCGACUUUACACAC-dTdT-3'. LipofectAMINEplus (Invitrogen) was used to transfect HBMECs overnight with cav-1 siRNA-1 (40 nM) and with cav-1 siRNA-2 (40 nM) for an additional $5 \mathrm{~h}$.

Western blotting. HMBEC membrane fractions were isolated as described by Gong et al. (1997), and total cell extracts were prepared using a standard procedure (Koon et al., 2006). Polyclonal antibodies against phospho-mitogen-activated protein kinase kinase (MEK) $\left(\operatorname{Ser}^{217 / 221}\right)$, MEK1/2, and extracellular signal-regulated kinase $1 / 2$ (ERK1/2) were from Cell Signaling Technology. Caveolin-1 polyclonal antibody was from Affinity BioReagents. Antibodies against ZO-1 and occludin were from Zymed Laboratories. Antibodies against phospho-ERK1/2 
and ZO-2 were from Santa Cruz Biotechnology. Pan-Ras monoclonal antibody was from BD Biosciences, and anti-actin antibody was purchased from Sigma-Aldrich. Immunoreactive bands were visualized by using horseradish peroxidase-conjugated secondary antibodies (Santa Cruz) and developed by ECL (GE Healthcare). The density of bands was calculated using the Scion Image program (Scion). Coimmunoprecipitation of caveolin-1 and Ras protein was performed according to a previously published technique (András et al., 2005) using anti-Ras antibody for immunoprecipitation and anti-caveolin-1 antibody for Western blotting.

Caveolae preparation. Cellular fractions were prepared using a detergent-free method described by Smart et al. (1995). In addition to caveolae membranes, postnuclear supernatant, plasma membranes, and intracellular membranes were also isolated during this procedure.

Immunofluorescence microscopy. Brain microvascular cells isolated from cav-1 ${ }^{-1-}$ and control mice were grown on slides coated with a mixture of collagen type I and IV and treated with Tat. ZO-1 immunoreactivity was detected as described previously (András et al., 2003). The slides were mounted using antifade reagent containing 4',6-diamidino-2-phenylindole (DAPI) (Invitrogen) to stain nuclear DNA. Samples were evaluated under the epifluorescence microscope.

Statistical analysis. Each experiment was repeated a minimum of three times. Data were expressed as mean \pm SEM. One-way or two-way ANOVA was used to compare mean responses among the treatments. Statistical probability of $p<0.05$ was considered significant.

\section{Results}

\section{Tat stimulates Ras activation}

Tat can interact with G-protein-coupled receptors, such as vascular endothelial growth factor receptor 2 (VEGFR-2) (András et al., 2005). Activation of these cell surface receptors may lead to stimulation of small GTPases, including the Ras signaling cascade. Ras activation is connected to the transformation of GDPRas to GTP-Ras. Therefore, we determined GTP-Ras levels in response to Tat exposure (Fig. $1 A, B$ ) using the pull-down assay. As illustrated in Figure $1 A$, exposure to $100 \mathrm{~nm}$ Tat resulted in a rapid and time-dependent increase in GTP-Ras. Indeed, GTPRas levels were elevated as the result of a 3 min treatment with Tat and returned to the control levels in cells exposed to Tat for 30 min or $3 \mathrm{~h}$. The total Ras level was not affected by Tat exposure. In addition, treatment with negative controls, such as bovine serum albumin (BSA) or immunoabsorbed Tat (AA-Tat) did not alter GTP-Ras levels in HBMECs. The effects of Tat on GTP-Ras levels were dose dependent, and a marked increase was observed in HBMECs exposed to $20 \mathrm{~nm}$ Tat. However, a maximum activation of Ras ( $\sim 2.4$-fold increase over basal values) was observed in cells treated with Tat at the concentration of $100 \mathrm{~nm}$ (Fig. $1 B$ ).

Next, we evaluated the expression of Ras in the membrane fraction of Tat-treated HBMECs. Confluent cultures were exposed to $100 \mathrm{~nm}$ Tat for up to $6 \mathrm{~h}$ and membrane Ras protein was analyzed by immunoblotting. As shown in Figure $1 C$, an increase in Ras in the membrane fraction occurred as early as 1 min after Tat exposure and was preserved for up to $60 \mathrm{~min}$. These effects were specific because AA-Tat did not affect membrane Ras levels. Similar to the results presented in Figure 1, $A$ and $B$, total Ras was not affected by Tat exposure.
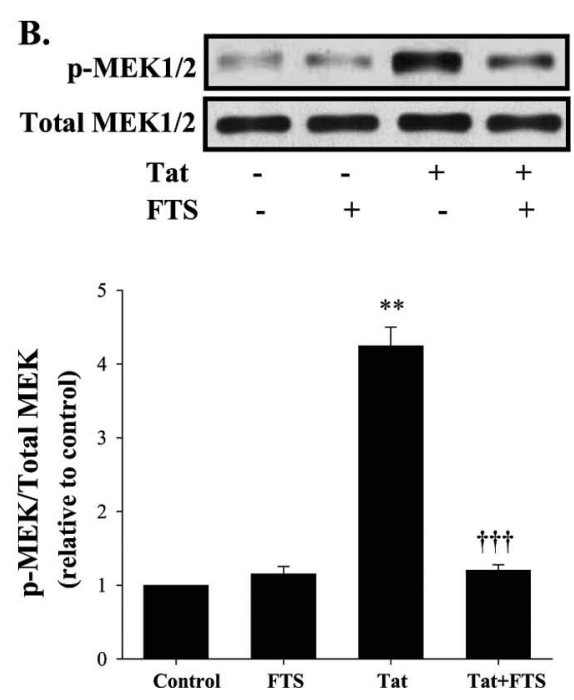

Figure 2. Tat-mediated activation of MEK1/2 in HBMECs is Ras dependent. $\boldsymbol{A}$, Time course analysis of MEK1/2 activation in $\boldsymbol{A}$. In and $\boldsymbol{B}$, the blots reflect representative data from three different experiments, and the bar graphs represent quantified results (mean $\pm S D$ ) from these experiments as analyzed by densitometry. ${ }^{* *} p<0.01$ compared with controls. ${ }^{\mathrm{tt}}$ Values in the group Tat + FTS are statistically different from those in the Tat group at $p<0.001$.

Tat activates downstream kinases of the Ras signaling cascade To evaluate the downstream signaling effects of Tat-induced activation of Ras, we determined the levels of phosphorylated MEK1/2 and ERK1/2 in Tat-treated HBMECs. The experiments were performed using confluent cultures exposed to $100 \mathrm{~nm}$ Tat for up to $3 \mathrm{~h}$. As shown in Figure 2A, exposure to Tat resulted in induction of MEK1/2 phosphorylation with a peak at $30 \mathrm{~min}$ ( $\sim 6.5$-fold increase), followed by a decline at $3 \mathrm{~h}$ after treatment. BSA, which was used as a negative control, did not have any effect on MEK1/2 phosphorylation. To confirm that the Ras pathway is involved in Tat-induced MEK1/2 activation, HBMECs were pretreated with farnesylthiosalicylic acid (FTS) $(20 \mu \mathrm{M})$, a specific inhibitor of Ras, before treatment with Tat. Figure $2 B$ indicates that preincubation with FTS efficiently blocked Tat-stimulated MEK1/2 phosphorylation. The total levels of MEK1/2 were not affected by Tat treatment.

Treatment with Tat significantly upregulated levels of phosphorylated ERK1/2 ( 3.7-fold increase at $30 \mathrm{~min}$ ) (Fig. $3 A$ ). Similar to MEK1/2 activation, these effects were also the most pronounced after $30 \mathrm{~min}$ of Tat exposure. Tat-induced activation of ERK1/2 was markedly blocked by the Ras inhibitor FTS (Fig. $3 B$ ) and MEK1/2 inhibitor 1,4-diamino-2,3-dicyano-1,4-bis (oaminophenylmercapto)butadiene (U0126) (0.6 $\mu \mathrm{M})$ (Fig. 3C), indicating that Tat-induced activation of ERK1/2 is a downstream effect of MEK1/2 and Ras stimulation.

\section{Tat upregulates caveolin-1 expression}

We hypothesize that caveolin-1 may play a regulatory role in Tat-mediated alteration of caveolae-associated signaling and expression of tight junction proteins. Before performing the experiments that directly address this hypothesis, we determined the effects of Tat on cellular caveolin-1 levels. Treatment of confluent HBMEC cultures with Tat resulted in a dose- and time-dependent elevation of membrane caveolin-1 levels (Fig. 4A, $B$, respectively). The highest elevation of caveolin-1 levels were observed in cells exposed to $100 \mathrm{~nm}$ Tat for $3 \mathrm{~min}$ ( $\sim 2.5$-fold compared with control). 
A.

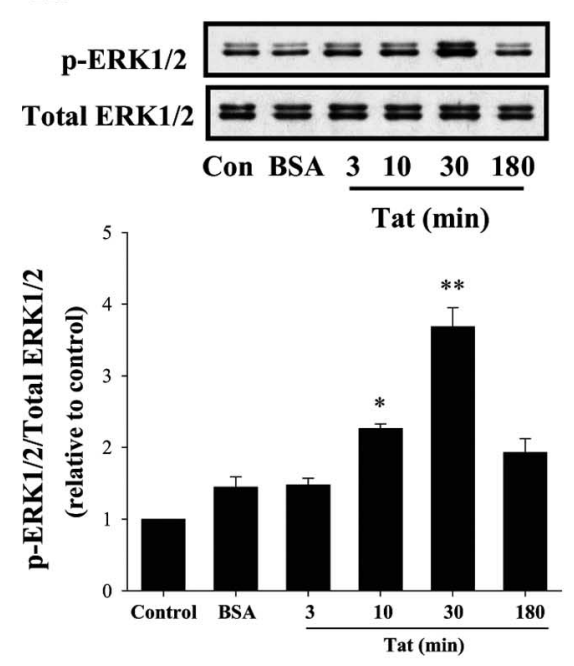

B.
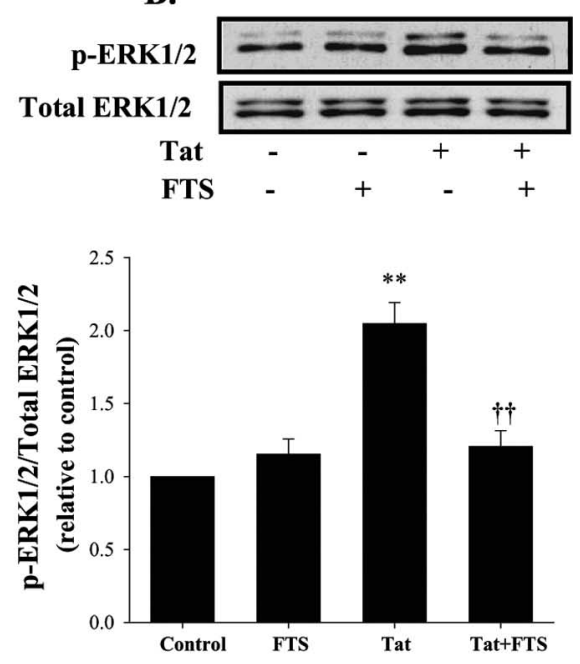

\section{C.}
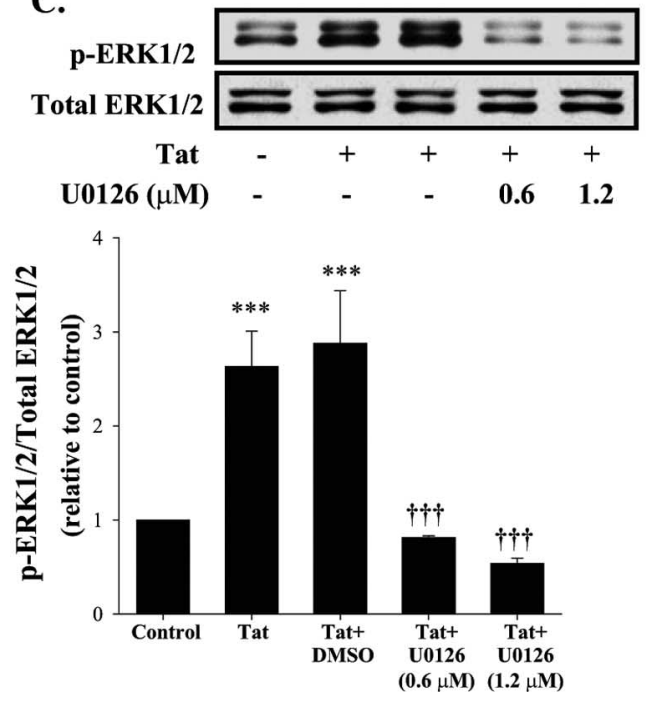

Figure 3. Tat-mediated activation of ERK1/2 in HBMECs is downstream from Ras and MEK1/2. $A$, Time course analysis of ERK1/2 activation induced by Tat. HBMECs were treated with $100 \mathrm{~nm}$ Tat for the indicated time periods. Phosphorylated (pERK1/2) and total ERK1/2 were analyzed by immunoblotting with specific antibodies. BSA was used as a negative control. $\boldsymbol{B}$, HBMECs were pretreated for $2 \mathrm{~h}$ with Ras inhibitor FTS $(20 \mu \mathrm{m})$ and exposed to $100 \mathrm{~nm}$ Tat for $30 \mathrm{~min}$. Phosphorylated and total ERK $1 / 2$ were determined as described in $A$. C, HBMECs were pretreated with the indicated concentrations of MEK $1 / 2$ inhibitor U0126, followed by exposure to $100 \mathrm{~nm}$ Tat for $30 \mathrm{~min}$. Phosphorylated and total ERK1/2 were analyzed as in $A$. In $A-C$, the blots reflect representative data from three different experiments, and the bar graphs represent quantified results (mean $\pm S D$ ) from these experiments as analyzed by densitometry. ${ }^{*} p<0.05,{ }^{* *} p<0.01,{ }^{* * *} p<0.001$ compared with controls; values in the group Tat + FTS or Tat + U0126 are statistically different from those in the Tat group at ${ }^{\mathrm{tt}} p<0.01$ or ${ }^{\mathrm{tt} t} p<0.001$.

However, a lower dose of Tat, such as $20 \mathrm{nM}$, also increased membrane caveolin- 1 levels. The specificity of these effects was determined by treatment with AA-Tat.

In time-dependent experiments, HBMECs were exposed to $100 \mathrm{~nm}$ Tat for up to $6 \mathrm{~h}$. Upregulation of membrane caveolin-1 was evident as early as $1 \mathrm{~min}$, peaked at $3 \mathrm{~min}$ (approximately threefold increase), and was preserved for up to $30 \mathrm{~min}$ of Tat exposure. Longer treatment times with Tat did not reach statistical significance (Fig. $4 B$ ). The effects of Tat were limited to the membrane fraction of caveolin-1 and total caveolin-1 levels were not altered independent of the treatment dose (Fig. 4A) or exposure time (Fig. $4 B$ ).
Caveolin-1 silencing abolishes Tat-

induced Ras activation

In the next series of experiments, we determined the role of caveolin-1 in Tat-mediated activation of Ras. First, we determined by coimmunoprecipitation that caveolin-1 interacts with Ras proteins. In the experiments presented in Figure 5A, immunoprecipitation was performed using anti-pan Ras antibody, followed by Western blotting with anti-caveolin-1 antibody. Similar results were obtained using a reverse combination of these antibodies (data not shown). Then, using a mixture of two specific caveolin-1 siRNAs, we silenced caveolin-1 protein expression by $\sim 70 \%$ compared with mock transfected control cultures. Transfection with the same amount of control siRNA did not affect caveolin-1 expression. In addition, actin expression in the cell lysates remained unchanged in transfected cells (Fig. 5B).

As indicated in Figure $5 C$, silencing of caveolin-1 effectively protected against Tat-induced Ras activation. In these experiments, HBMECs were treated with $100 \mathrm{~nm}$ Tat for $3 \mathrm{~min}$. Both Tat treatment and caveolin-1 silencing did not alter the total Ras levels in HBMECs. These results provide evidence that caveolin-1 is an upstream modulator of Ras signaling in HBMECs.

Tat alters expression of tight junction proteins in caveolae through the Ras-mediated signaling

The integrity of tight junctions is the main factor that determines the integrity and the barrier function of the brain endothelium. The functional state of tight junctions depends on phosphorylation processes; therefore, we evaluated the involvement of the Ras signaling and caveolin-1 in Tatmediated alterations of tight junction protein expression.

Exposure to Tat (100 nM) markedly diminished the expression of several tight junction proteins, such as occludin, ZO-1, and $\mathrm{ZO}-2$, in HBMECs as analyzed by Western blotting (Fig. 6A). These effects were induced in cells treated with Tat for $12 \mathrm{~h}$ and persisted $24 \mathrm{~h}$ after adding Tat to cell cultures. Shorter exposure times or control treatment with AA-Tat did not alter the expression of tight junction proteins. Actin was determined as a housekeeping protein and its levels were not changed in response to Tat or AA-Tat.

To further determine the relationship between tight junction proteins and caveolae, we used a detergent-free method for preparation of caveolae-rich cellular fractions (Smart et al., 1995). As indicated in Figure $6 \mathrm{~B}$, the basal expression of tight junction proteins is enriched in the caveolae fraction of HBMECs compared with other cellular components, including plasma membranes. Most importantly, Tat-mediated alterations of occludin, ZO-1, and ZO-2 expression were specifically localized to the 
caveolae fraction of HBMECs. Statistical analysis of these effects is presented in Figure $6 C$. Densitometry assessment revealed that ZO-1, ZO-2, and occludin expression were reduced by $47.5,58.6$, and $49.7 \%$, respectively, in the caveolae fraction of $\mathrm{HB}$ MECs treated with Tat for $15 \mathrm{~h}$. Figure $6 \mathrm{C}$ also indicates that the effects of Tat on the expression of tight junction proteins are regulated by the Ras signaling. Indeed, pretreatment of HBMECs with $5 \mu \mathrm{M}$ FTS for 3 h completely inhibited Tat-induced alterations of occludin, ZO-1, and ZO-2 expression in the caveolae fraction of $\mathrm{HB}$ MECs. Treatment with FTS alone did not affect expression of tight junction proteins.

Lack of caveolin-1 attenuates Tatinduced alterations of tight junction protein expression and redistribution Strong expression of tight junction proteins in the caveolae fraction of HBMECs prompted us to determine the effects of caveolin-1 silencing on Tat-induced tight junction protein expression. As indicated in Figure $7 A$, knocking down caveolin-1 expression did not affect the basal expression of tight junction proteins in untreated HBMECs. However, caveolin-1 silencing effectively protected against Tat-induced diminished the expression of occludin, ZO-1, and ZO-2. These effects were next confirmed in brain endothelial cells isolated from mice lacking caveolin-1 (cav-1 $1^{-1-}$ mice) (Fig. $7 B$ ). Tat treatment $(100 \mathrm{nM} ; 15 \mathrm{~h})$ of brain microvascular endothelial cells isolated from control mice resulted in diminished and fragmented expression of ZO-1 immunoreactivity at the cell-cell borders (longer arrows) and redistribution of $\mathrm{ZO}-1$ from the cell borders into the cytoplasm (shorter and open arrows). Note that the experiments were performed on confluent cultures as illustrated by uniformly distributed DAPI staining for nuclear DNA. Importantly, Tat-mediated effects on ZO-1 immunoreactivity were markedly attenuated in brain microvascular endothelial cells isolated from cav-1 ${ }^{-/-}$mice.

\section{Discussion}

Lipid rafts and caveolae play an important role in interactions of host cells with infectious agents, including enveloped viruses, such as HIV-1 (Suzuki and Suzuki, 2006). By increasing the spatial density of cell surface receptors, lipid rafts enhance the access of pathogens to cells and aid the cellular entry of infection agents. In addition, lipid rafts can direct pathogen trafficking to specific sites for replication and/or facilitate the assembly of the enveloped viruses during replication. It is known that lipid rafts play important roles in HIV-1 infection. It was demonstrated that HIV-1 binding to T-cells involves interactions between HIV-1 envelope proteins gp120-gp41 with raft-associated CD4 and coreceptors CCR5 and CXCR4. Disruption of lipid rafts by cholesterol depletion or by blocking sphingomyelin synthesis inhibits HIV entry into T-cells. Rafts contribute also to HIV assembly and budding. Specifically, HIV-1 proteins Gag and Env, which mediate several steps in virus assembly, are anchored into T-cell lipid rafts (Huang et al., 2007). The results of the present study provide strong evidence that caveolae play a regulatory role in activation of HBMECs and disruption of tight junction proteins in response to cellular exposure to viral proteins, such as HIV-1 Tat.

The mechanisms of Tat interaction with the cell surface, including lipid rafts and caveolar membranes, are not fully understood. It was suggested that Tat can mimic extracellular matrix proteins and bind to $\alpha \mathrm{v} \beta 3$ and $\alpha 5 \beta 1$ integrins through the arginine-glycine-aspartic domain (Toschi et al., 2006). In addition, the VEGFR-2 has been proposed to serve as a high-affinity receptor for Tat in endothelial cells (Albini et al., 1996). This notion was supported by our data that indicate that blocking VEGFR-2 can attenuate Tat-induced disruption of tight junctions (András et al., 2005). VEGFR-2 and a variety of other cell surface receptors that belong to the large family of G-proteincoupled receptors are localized to caveolae in endothelial cells. Activation of these receptors can stimulate the heterotrimeric G-proteins, which then induce the Ras signaling (Hancock, 2003; Wennerberg et al., 2005). Thus, interaction of Tat with cell surface receptors, such as VEGFR-2, can be directly responsible for an early activation of Ras signaling as observed in the present study (Fig. 1). In support of this notion, it was shown that Tat binding to cell membranes resulted in activation of the Ras/ MAPK (mitogen-activated protein kinase) signaling pathway and contributed to cell progression through the $G_{1}$ phase in response to mitogen in primary and immortalized human umbilical endothelial cells (Toschi et al., 2006). Our present data also confirms that Tat-induced activation of Ras results in stimulation of the downstream kinases, such as MEK1/2 and ERK1/2 (Figs. 2, 3).

In addition to activation of the Ras cascade, treatment with Tat upregulated membrane caveolin-1 protein levels in HBMECs. These results indicate a close interaction of Tat with caveolae. The effects of Tat on caveolin-1 levels were dose dependent (Fig. 4A) and occurred within minutes of Tat exposure (Fig. $4 B$ ). To explain such an early response, it should be pointed out that caveolin proteins are present not only in caveolae but also in the trans-Golgi network and in caveosome, endocytic organelles 
A.

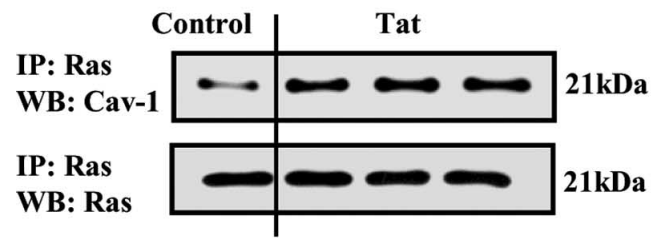

B.

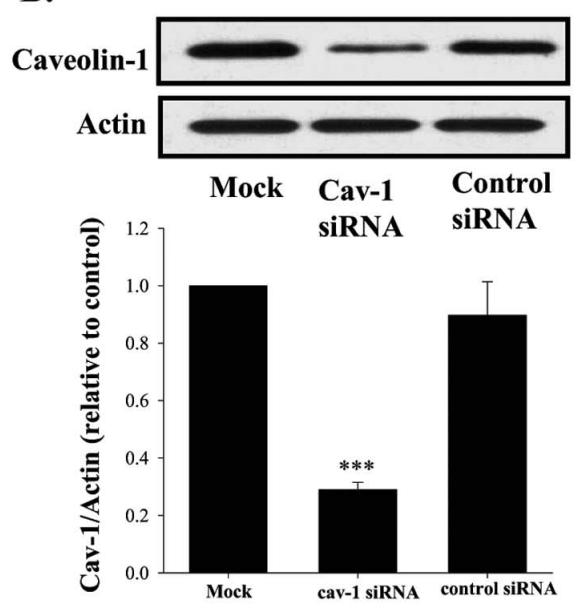

C.

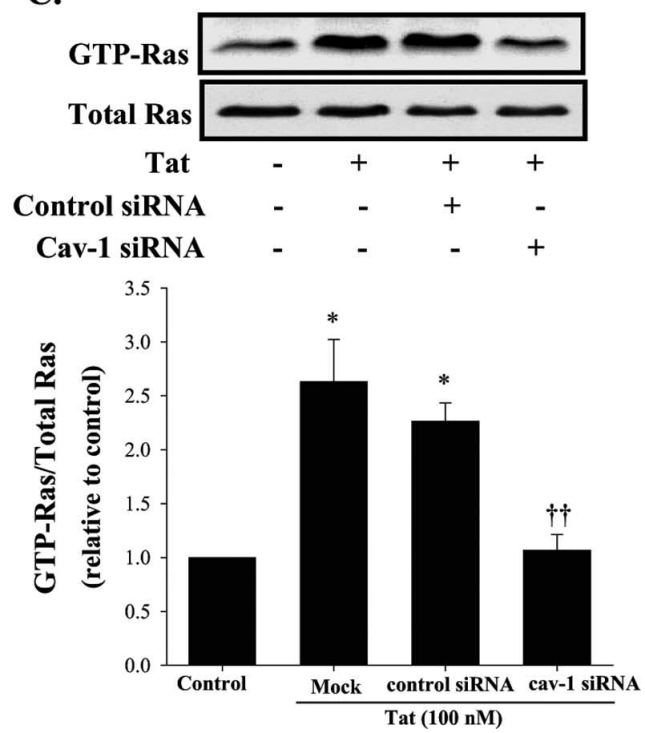

Figure 5. Tat-mediated activation of Ras is regulated by caveolin-1.A, Ras coimmunoprecipitates with caveolin-1. Lysates of control and Tat-treated HBMEC cultures were immunoprecipitated using monoclonal pan-Ras antibody, followed by Western blotting with anti-caveolin-1 antibody or antipan-Ras antibody (control). IP, Immunoprecipitation; WB, Western blotting. $\boldsymbol{B}$, HBMECs were transfected with specific caveolin-1 siRNA or with control siRNA and caveolin-1 levels were analyzed by immunoblotting in total cell extracts. The silencing procedure resulted in $\sim 70 \%$ decrease in cellular caveolin-1 levels. C, Caveolin-1 was silenced in HBMECs as in A, followed by treatment with Tat (100 $\mathrm{nm)} \mathrm{for} 3 \mathrm{~min}$. Active GTP-Ras and total Ras were determined as in Figure 1. The blots reflect representative data from four different experiments, and the bar graphs represent quantified results (mean \pm SD) from these experiments as analyzed, by densitometry. ${ }^{*} p<0.05$, ${ }^{* * *} p<0.001$ compared with controls or mock transfection. ${ }^{+1}$ Data in the Tat + cav-1 siRNA are significantly different from those in the Tat group at $p<0.01$. that contain multiple caveolar domains. Caveolar domains present in the Golgi complexes actively detach as vesicles distinct from the trans-Golgi network. It was estimated that $\sim 100-200$ caveolae can move to the plasma membrane every minute in CV-1 cells (Pelkmans et al., 2001; Pelkmans and Helenius, 2002; Tagawa et al., 2005). Because caveolae are abundant in HBMECs, membrane levels of caveolin-1 can also rapidly change in response to Tat treatment. Similarly, a dramatic enhancement of a mobile caveolae fraction was shown in response to SV40 (simian virus 40) (Tagawa et al., 2005).

Another novel finding reported in the present study is the observation that caveolin-1 can regulate Tat-induced disruption of tight junction proteins in HBMECs. These results are important because alterations of tight junctions can directly influence the integrity of the BBB and facilitate the entry of HIV-1-infected cells into the brain. In addition, the integrity of the brain microvasculature is critical to maintain brain homeostasis and viability of neurons (Hawkins and Davis, 2005; Abbott et al., 2006). In the present study, we focused on the regulation of occludin, $\mathrm{ZO}-1$, and ZO-2 expression. Occludin is a $60-65 \mathrm{kDa}$ transmembrane protein that is capable of linking with $\mathrm{ZO}-1$. The main functions of occludin are regulation and sealing of tight junctions. In fact, vesicular removal of occludin from tight junctions correlated with loss of barrier function (Lacaz-Vieira et al., 1999; Abbott et al., 2006). ZO-1 and ZO-2 are cytoplasmic proteins that belong to the family of membrane-associated guanylate kinases. They also play a role in signal transduction mechanisms and in anchoring the transmembrane tight junction proteins to the cytoskeleton (Wolburg and Lippoldt, 2002).

Disruption of the integrity of endothelial monolayers in response to HIV-1 has been described previously (Dallasta et al., 1999; Boven et al., 2000; Persidsky et al., 2006). Clinical studies revealed the absence or fragmentation of occludin and $\mathrm{ZO}-1$ in HIV-positive patients with encephalitis or HIV-1-associated dementia (Dallasta et al., 1999; Boven et al., 2000). To confirm the role of tight junctions in HIV-1-associated brain pathology, it was shown that monocyte infiltration was associated with the disruption of ZO-1 immunoreactivity in HIV-1-positive patients (Boven et al., 2000). Several HIV-1 proteins, such as gp120, Nef, and Tat were described to alter the expression of tight junction proteins in vitro and to enhance transendothelial migration of monocytes (Annunziata, 2003). We reported that the exposure of primary brain endothelial cells to Tat resulted in redistribution and decreased expression of several tight junction proteins, including claudin-5, ZO-1, and ZO-2 (András et al., 2003, 2005). These effects are regulated at the transcriptional levels (András et al., 2005), which may explain a temporal delay between a rapid activation of signaling processes and alterations of tight junction protein expression as observed in the present study. Indeed, we observed that Tat induced the Ras cascade and altered membrane caveolin-1 levels within minutes of cellular exposure. However, longer exposure times, such as 12 or $24 \mathrm{~h}$, were required to induce tight junction changes.

The present study describes novel molecular mechanisms of Tat-induced alterations of tight junction protein expression via caveolae-associated signaling. Our immunoblotting results indicate that caveolae are enriched with tight junction proteins compared with other cellular fractions. Moreover, Tat-induced changes in occludin, ZO-1, and ZO-2 expression appear to be exclusively limited to caveolae (Fig. 6). These results are in agreement with our unpublished observations that specific tight junction proteins are associated with caveolae and can be immunoprecipitated with caveolin-1. Previous reports also indicated that 
tight junction proteins are present in lipidrich membrane microdomains with biophysical characteristics of detergent insoluble glycolipids, and the association of caveolin-1 with occludin was visualized by high-frequency imaging and morphometric analyses (Nusrat et al., 2000).

To further demonstrate the relationship between tight junctions and functional caveolae, our results demonstrate that silencing of caveolin-1 can protect against diminished tight junction protein expression in Tat-treated HBMECs (Fig. $7 A$ ). Caveolin-1 silencing does not result in a complete elimination of protein expression, leaving $\sim 30 \%$ of normal caveolin-1 levels (Fig. 5B). Therefore, we crosschecked the effects of caveolin-1 silencing on tight junction protein expression using brain microvascular endothelial cells isolated from caveolin-1-deficient mice and from the corresponding controls. As shown in Figure $7 B$, lack of caveolin-1 markedly protected against Tat-induced disruption of ZO-1 immunoreactivity. These observations are further supported by the report that disruption of caveolae by cholesterol extraction from plasma membrane prevented both barrier dysfunction and occludin redistribution after actin depolymerization (Shen and Turner, 2005).

The protective effects of caveolin-1 silencing appear to be related to modulation of the Ras cascade. Indeed, silencing of caveolin-1 effectively prevented Tatmediated activation of Ras (Fig. 5), the signaling pathway that regulates the expression of tight junction proteins. However, several recent reports indicated that cellular uptake of Tat may occur via caveolar-associated mechanisms (Ferrari et al., 2003; Fittipaldi et al., 2003; Jones et al., 2005; Renigunta et al., 2006). This fact may suggest that the protective effects of caveolin-1 silencing might also be related to disruption of caveolae and diminished internalization of Tat. However, pharmacological inhibition of the Ras pathway (Fig. 6) mimicked the protective effects of caveolin-1 silencing on Tat-induced alterations of tight junction protein expression (Fig. $7 A$ ), indicating the specificity of the observed effects. Thus, caveolin-1 may constitute an early and critical modulator that controls signaling pathways leading to the disruption of tight junction proteins.

In conclusion, the present study demonstrates that exposure to Tat results in activation of the Ras signaling, upregulation of caveolin-1, and disruption of tight junction proteins (occludin, ZO-1, and ZO-2). These events are interrelated, because inhibition of the Ras pathway protected against Tatinduced disruption of tight junction proteins. In addition, caveolin-1 silencing blocked both Tat-mediated activation of Ras and alterations of tight junction proteins. Thus, Tatinduced caveolin-1 upregulation may constitute an early event that can initiate signaling transduction pathway, leading to the disruption of tight junctions, and HIV-1 passage across the brain endothelium.
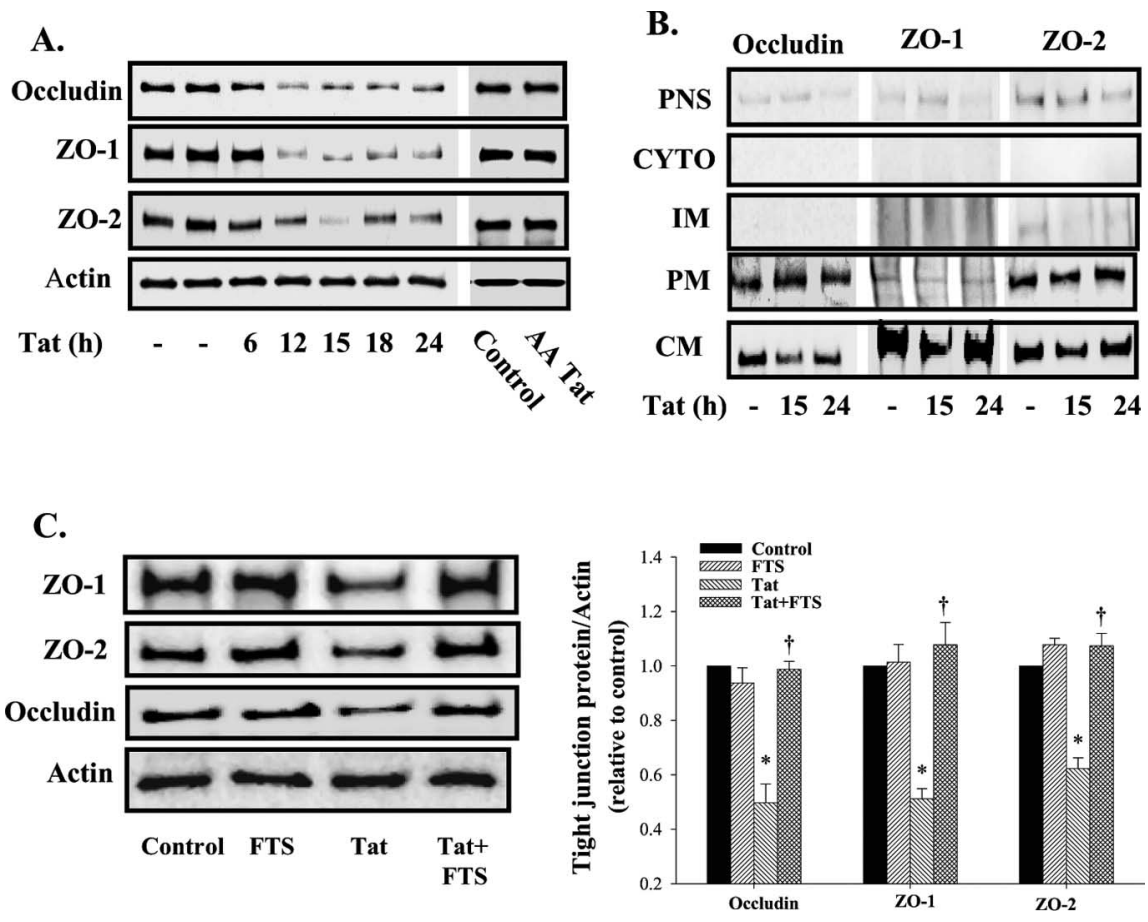

Figure 6. Exposure to Tat alters expression of tight junction proteins in caveolae fraction of HBMECs via the Ras-regulated motel of tight junction proteins was detected by immunoblotting in postnuclear soup (PNS), cytosol (CYTO), F F for $3 \mathrm{~h}$, and then incubated with $100 \mathrm{~nm}$ Tat for $15 \mathrm{~h}$. FTS was retained in cell culture medium for the duration of Tat 作 protein and loading control. The blots reflect representative data from four different experiments, and the bar graph represent quantified results (mean $\pm S D$ ) from these experiments as analyzed by densitometry. ${ }^{*} p<0.05$ compared with control (mock transfection). ${ }^{\dagger}$ Data in the Tat+FTS are significantly different from those in the Tat group at $p<0.05$.

\section{References}

Abbott NJ, Rönnbäck L, Hansson E (2006) Astrocyte-endothelial interactions at the blood-brain barrier. Nat Rev Neurosci 7:41-53.

Albini A, Soldi R, Giunciuglio D, Giraudo E, Benelli R, Primo L, Noonan D, Salio M, Camussi G, Rockl W, Bussolino F (1996) The angiogenesis induced by HIV-1 tat protein is mediated by the Flk-1/KDR receptor on vascular endothelial cells. Nat Med 2:1371-1375.

András IE, Pu H, Deli MA, Nath A, Hennig B, Toborek M (2003) HIV-1 Tat protein alters tight junction protein expression and distribution in cultured brain endothelial cells. J Neurosci Res 74:255-265.

András IE, Pu H, Tian J, Deli MA, Nath A, Hennig B, Toborek M (2005) Signaling mechanisms of HIV-1 Tat-induced alterations of claudin-5 expression in brain endothelial cells. J Cereb Blood Flow Metab 25:1159-1170

Annunziata P (2003) Blood-brain barrier changes during invasion of the central nervous system by HIV-1. Old and new insights into the mechanism. J Neurol 250:901-906.

Banks WA, Ercal N, Price TO (2006) The blood-brain barrier in neuroAIDS. Curr HIV Res 4:259-266.

Boven LA, Middel J, Verhoef J, De Groot CJ, Nottet HS (2000) Monocyte infiltration is highly associated with loss of the tight junction protein zonula occludens in HIV-1-associated dementia. Neuropathol Appl Neurobiol 26:356-360.

Cohen AW, Hnasko R, Schubert W, Lisanti MP (2004) Role of caveolae and caveolins in health and disease. Physiol Rev 84:1341-1379.

Dallasta LM, Pisarov LA, Esplen JE, Werley JV, Moses AV, Nelson JA, Achim CL (1999) Blood-brain barrier tight junction disruption in human immunodeficiency virus-1 encephalitis. Am J Pathol 155:1915-1927.

Dykstra M, Cherukuri A, Sohn HW, Tzeng SJ, Pierce SK (2003) Location is everything: lipid rafts and immune cell signaling. Annu Rev Immunol 21:457-481. 

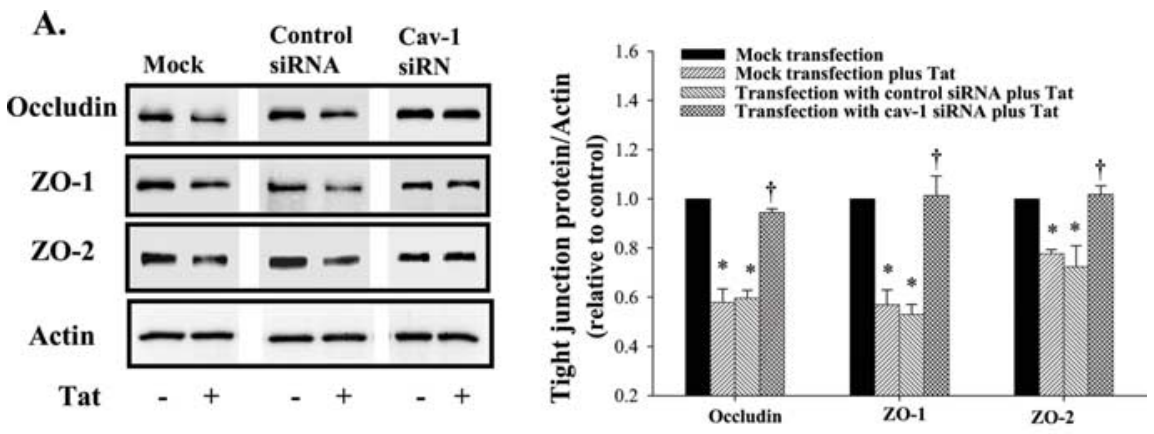

B.

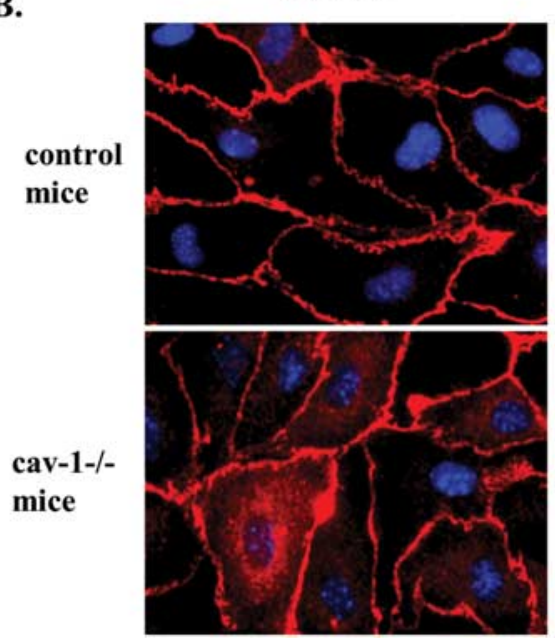

Control

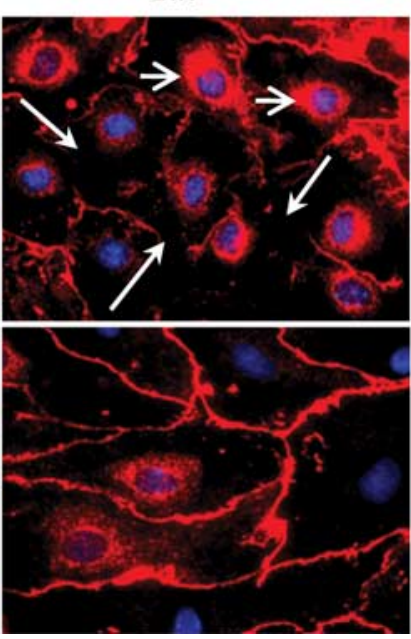

Figure 7. Caveolin-1 modulates Tat-induced alterations of tight junction protein expression. $\boldsymbol{A}$, Caveolin-1 was silenced in HBMECs as in Figure 5, followed by exposure to $100 \mathrm{~nm}$ Tat for $15 \mathrm{~h}$. Expression of tight junction proteins (occludin, ZO-1, and ZO-2) was analyzed by immunoblotting in total cell extracts. Control experiments include mock transfection and transfection with control siRNA. The blots reflect representative data from three different experiments. ${ }^{*} p<0.05$ compared with control (mock transfection). ${ }^{\dagger}$ Data in the cultures exposed to Tat and caveolin-1 siRNA are significantly different from those in the cultures exposed to Tat alone or Tat plus control siRNA at $p<0.05$. B , Brain microvascular endothelial cells were isolated from cavoelin1-deficient $\left(\mathrm{cav}-1^{-1-}\right)$ mice and the respective controls. Cells were cultured until confluence and treated with $100 \mathrm{~nm}$ Tat for $15 \mathrm{~h}$. Z0-1 immunoreactivity was determined by immunofluorescence (red staining). In addition, DAPI staining was performed to visualize the nuclei (blue staining). Tat treatment resulted in diminished and fragmented expression of 20-1 immunoreactivity at the cell-cell borders (longer arrows) and redistribution of Z0-1 from the cell borders into the cytoplasm (shorter and open arrows) in cells from control mice. These effects were markedly attenuated in cells from cav $-1^{-1-}$ mice. The images were taken using a $60 \times$ objective and a $10 \times$ ocular lens.

Eugenin EA, King JE, Nath A, Calderon TM, Zukin RS, Bennett MV, Berman JW (2007) HIV-tat induces formation of an LRP-PSD-95- NMDARnNOS complex that promotes apoptosis in neurons and astrocytes. Proc Natl Acad Sci U S A 104:3438-3443.

Ferrari A, Pellegrini V, Arcangeli C, Fittipaldi A, Giacca M, Beltram F (2003) Caveolae-mediated internalization of extracellular HIV-1 tat fusion proteins visualized in real time. Mol Ther 8:284-294.

Fittipaldi A, Ferrari A, Zoppé M, Arcangeli C, Pellegrini V, Beltram F, Giacca M (2003) Cell membrane lipid rafts mediate caveolar endocytosis of HIV-1 Tat fusion proteins. J Biol Chem 278:34141-34149.

Furuse M, Hirase T, Itoh M, Nagafuchi A, Yonemura S, Tsukita S, Tsukita S (1993) Occludin: a novel integral membrane protein localizing at tight junctions. J Cell Biol 123:1777-1788.

Gong MC, Fujihara H, Somlyo AV, Somlyo AP (1997) Translocation of rhoA associated with $\mathrm{Ca}^{2+}$ sensitization of smooth muscle. J Biol Chem 272:10704-10709.

González-Scarano F, Martín-García J (2005) The neuropathogenesis of AIDS. Nat Rev Immunol 5:69-81.
Hancock JF (2003) Ras proteins: different signals from different locations. Nat Rev Mol Cell Biol 4:373-384.

Hawkins BT, Davis TP (2005) The blood-brain barrier/neurovascular unit in health and disease. Pharmacol Rev 57:173-185.

Huang JH, Lu L, Lu H, Chen X, Jiang S, Chen YH (2007) Identification of the HIV-1 gp41 corebinding motif in the scaffolding domain of caveolin-1. J Biol Chem 282:6143-6152.

Jones SW, Christison R, Bundell K, Voyce CJ, Brockbank SM, Newham P, Lindsay MA (2005) Characterisation of cell-penetrating peptide-mediated peptide delivery. Br J Pharmacol 145:1093-1102.

Koon HW, Zhao D, Zhan Y, Rhee SH, Moyer MP, Pothoulakis C (2006) Substance P stimulates cyclooxygenase- 2 and prostaglandin E2 expression through JAK-STAT activation in human colonic epithelial cells. J Immunol 176: 5050-5059.

Lacaz-Vieira F, Jaeger MM, Farshori P, Kachar B (1999) Small synthetic peptides homologous to segments of the first external loop of occludin impair tight junction resealing. J Membr Biol 168:289-297.

Ma M, Nath A (1997) Molecular determinants for cellular uptake of Tat protein of human immunodeficiency virus type 1 in brain cells. J Virol 71:2495-2499.

Nusrat A, Parkos CA, Verkade P, Foley CS, Liang TW, Innis-Whitehouse W, Eastburn KK, Madara JL (2000) Tight junctions are membrane microdomains. J Cell Sci 113:1771-1781.

Pelkmans L, Helenius A (2002) Endocytosis via caveolae. Traffic 3:311-320.

Pelkmans L, Kartenbeck J, Helenius A (2001) Caveolar endocytosis of simian virus 40 reveals a new two-step vesicular-transport pathway to the ER. Nat Cell Biol 3:473-483.

Persidsky Y, Poluektova L (2006) Immune privilege and HIV-1 persistence in the CNS. Immunol Rev 213:180-194.

Persidsky Y, Heilman D, Haorah J, Zelivyanskaya M, Persidsky R, Weber GA, Shimokawa H, Kaibuchi K, Ikezu T (2006) Rho-mediated regulation of tight junctions during monocyte migration across the blood-brain barrier in HIV-1 encephalitis (HIVE). Blood 107:4770-4780.

Pike LJ (2003) Lipid rafts: bringing order to chaos. J Lipid Res 44:655-667.

Rao R, Logan B, Forrest K, Roszman TL, Goebel J (2004) Lipid rafts in cytokine signaling. Cytokine Growth Factor Rev 15:103-110.

Razani B, Engelman JA, Wang XB, Schubert W, Zhang XL, Marks CB, Macaluso F, Russell RG, Li M, Pestell RG, Di Vizio D, Hou H Jr, Kneitz B, Lagaud G, Christ GJ, Edelmann W, Lisanti MP (2001) Caveolin-1 null mice are viable but show evidence of hyperproliferative and vascular abnormalities. J Biol Chem 276:38121-38138.

Renigunta A, Krasteva G, König P, Rose F, Klepetko W, Grimminger F, Seeger W, Hänze J (2006) DNA transfer into human lung cells is improved with Tat-RGD peptide by caveoli-mediated endocytosis. Bioconjug Chem 17:327-334.

Repetto S, Salani B, Maggi D, Cordera R (2005) Insulin and IGF-I phosphorylate eNOS in HUVECs by a caveolin-1 dependent mechanism. Biochem Biophys Res Commun 337:849-852.

Rumbaugh J, Turchan-Cholewo J, Galey D, St Hillaire C, Anderson C, Conant K, Nath A (2006) Interaction of HIV Tat and matrix metalloproteinase in HIV neuropathogenesis: a new host defense mechanism. FASEB J 20:1736-1738.

Shen L, Turner JR (2005) Actin depolymerization disrupts tight junctions via caveolae-mediated endocytosis. Mol Biol Cell 16:3919-3936. 
Smart EJ, Ying YS, Mineo C, Anderson RG (1995) A detergent-free method for purifying caveolae membrane from tissue culture cells. Proc Natl Acad Sci U S A 92:10104-10108.

Suzuki T, Suzuki Y (2006) Virus infection and lipid rafts. Biol Pharm Bull 29:1538-1541.

Tagawa A, Mezzacasa A, Hayer A, Longatti A, Pelkmans L, Helenius A (2005) Assembly and trafficking of caveolar domains in the cell: caveolae as stable, cargo-triggered, vesicular transporters. J Cell Biol 170:769-779.

Toborek M, Lee YW, Flora G, Pu H, András IE, Wylegala E, Hennig B, Nath A (2005) Mechanisms of the blood-brain barrier disruption in HIV-1 infection. Cell Mol Neurobiol 25:181-199.

Toschi E, Bacigalupo I, Strippoli R, Chiozzini C, Cereseto A, Falchi M, Nappi F, Sgadari C, Barillari G, Mainiero F, Ensoli B (2006) HIV-1 Tat regulates endothelial cell cycle progression via activation of the Ras/ERK MAPK signaling pathway. Mol Biol Cell 17:1985-1994.

Weiss JM, Nath A, Major EO, Berman JW (1999) HIV-1 Tat induces monocyte chemoattractant protein-1-mediated monocyte transmigration across a model of the human blood-brain barrier and up-regulates CCR5 expression on human monocytes. J Immunol 163:2953-2959.

Weksler BB, Subileau EA, Perrière N, Charneau P, Holloway K, Leveque M, Tricoire-Leignel H, Nicotra A, Bourdoulous S, Turowski P, Male DK, Roux F, Greenwood J, Romero IA, Couraud PO (2005) Blood-brain barrier-specific properties of a human adult brain endothelial cell line. FASEB J 19:1872-1874.

Wennerberg K, Rossman KL, Der CJ (2005) The Ras superfamily at a glance. J Cell Sci 118:843-846.

Westendorp MO, Frank R, Ochsenbauer C, Stricker K, Dhein J, Walczak H, Debatin KM, Krammer PH (1995) Sensitization of T cells to CD95mediated apoptosis by HIV-1 Tat and gp120. Nature 375:497-500.

Wolburg H, Lippoldt A (2002) Tight junctions of the blood-brain barrier: development, composition and regulation. Vascul Pharmacol 38:323-337.

Wu DT, Woodman SE, Weiss JM, McManus CM, D’Aversa TG, Hesselgesser J, Major EO, Nath A, Berman JW (2000) Mechanisms of leukocyte trafficking into the CNS. J Neurovirol 6 [Suppl 1]:S82-S85.

Wu RF, Gu Y, Xu YC, Mitola S, Bussolino F, Terada LS (2004) Human immunodeficiency virus type 1 Tat regulates endothelial cell actin cytoskeletal dynamics through PAK1 activation and oxidant production. J Virol 78:779-789.

Xiao H, Neuveut C, Tiffany HL, Benkirane M, Rich EA, Murphy PM, Jeang KT (2000) Selective CXCR4 antagonism by Tat: implications for in vivo expansion of coreceptor use by HIV-1. Proc Natl Acad Sci U S A 97: 11466-11471. 